This item was submitted to Loughborough's Research Repository by the author.

Items in Figshare are protected by copyright, with all rights reserved, unless otherwise indicated.

\title{
Spatially integrated speckle intensity: maximum resistance to decorrelation caused by in-plane target displacement
}

\section{PLEASE CITE THE PUBLISHED VERSION}

http://dx.doi.org/10.1364/AO.37.007062

\section{PUBLISHER}

(C) Optical Society of America

VERSION

VoR (Version of Record)

\section{LICENCE}

CC BY-NC-ND 4.0

\section{REPOSITORY RECORD}

Tullis, lain D.C., Neil A. Halliwell, and Steve Rothberg. 2019. "Spatially Integrated Speckle Intensity: Maximum Resistance to Decorrelation Caused by In-plane Target Displacement”. figshare.

https://hdl.handle.net/2134/8839. 
This item was submitted to Loughborough's Institutional Repository (https://dspace.lboro.ac.uk/) by the author and is made available under the following Creative Commons Licence conditions.

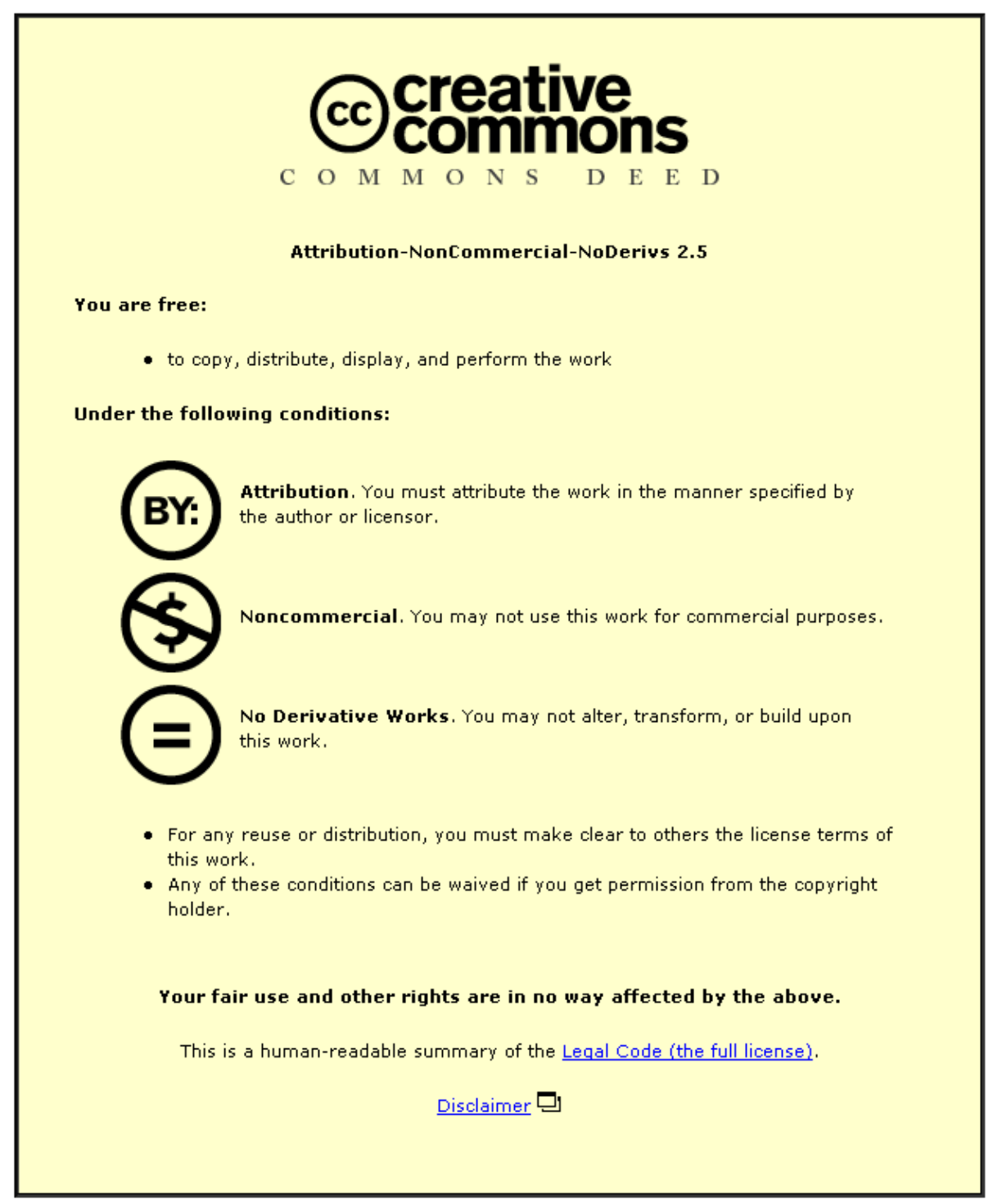

For the full text of this licence, please go to: http://creativecommons.org/licenses/by-nc-nd/2.5/ 


\title{
Spatially integrated speckle intensity: maximum resistance to decorrelation caused by in-plane target displacement
}

\author{
Iain D. C. Tullis, Neil A. Halliwell, and Steven J. Rothberg
}

\begin{abstract}
Laser speckle produced from a diffuse object can be used in determining the angular position of a rotating object. When the object rotates the backscattered speckle pattern, which changes continuously but repeats exactly with every revolution, is sampled by a suitably positioned photodetector. The photodetector output signal is periodic, and one period is stored in the memory as a reference. Shaft position can then be determined by the comparison of this stored reference signal with the current photodetector output signal. When the shaft is axially displaced, for example, by vibration, the backscattered speckle pattern changes on the photodetector and the similarity between the reference signal and the current signal is reduced. We examine the cross correlation of the real-time photodetector output signal and the stored reference signal as a function of axial shaft position. Use of a rotating shaft when collecting data is shown to be an efficient means by which to make effectively several thousand independent estimates of the maximum axial displacement tolerable before decorrelation of the photodetector output. Theoretical results and experiments conducted show that the decorrelation displacement varies, according to optical configuration, to a maximum value of 0.7 of the beam diameter. This has important implications for a proposed laser torquemeter as well as additional applications in which changes to the sampled speckle pattern, including decorrelation, are either desirable or undesirable. (c) 1998 Optical Society of America

OCIS codes: $\quad 030.6140,120.3930$.
\end{abstract}

\section{Introduction}

The random intensity pattern formed when coherent light is scattered from a rough surface is known as a speckle pattern. We describe a system in which a laser beam illuminates a small region on a rotating shaft, as shown in Fig. 1. A photodetector samples the backscattered speckle pattern and produces an output that is proportional to the speckle intensity integrated over its surface.

Figure 2 shows the sampled photodetector output (with the dc offset removed) from approximately one and a half revolutions of the rotating shaft. The correlation width of this signal is strongly influenced by the relative sizes of the speckles and the photodetector, and we address this issue theoretically. In

The authors are with the Department of Mechanical Engineering, Loughborough University, Loughborough, Leicestershire, LE11 3TV, United Kingdom.

Received 5 January 1998; revised manuscript received 18 June 1998.

0003-6935/98/307062-08\$15.00/0

(C) 1998 Optical Society of America the data shown approximately ten speckles were collected on the photodetector. The evident periodicity of the photodetector output in Fig. 2 is confirmed by its power spectrum, taken over 50 revolutions of the shaft, as shown in Fig. 3, in which the strong peaks occur at the rotation frequency and its harmonics.

When the target is rotating, the repeatability of the photodetector output signal is sensitive to motion of the target; for example, if the shaft vibrates axially ( $\xi$ direction), only a proportion of the original population of scatterers is illuminated and changes occur in the speckle pattern incident on the photodetector. In certain cases the speckles translate, moving as a whole without appreciable change in shape. In other cases the individual speckles boil, deforming, disappearing, and reappearing without appreciable translation. ${ }^{1}$ In either case the correlation between the photodetector output and the reference signal is reduced, with the rate at which this decorrelation occurs dependent on the speckle behavior.

We present the theory and supporting experimentation demonstrating how resistance to decorrelation caused by axial motion varies with the optical configuration. The aim of this study is to find optical con- 


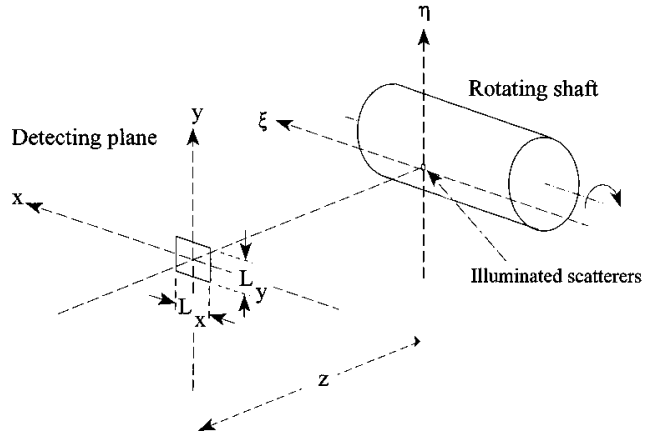

Fig. 1. Schematic diagram of the optical arrangement showing the coordinate axes.

figurations that maximize resistance to decorrelation of the photodetector output during axial vibration of a rotating shaft; however, the conclusions are equally applicable to decorrelation owing to the in-plane motion of any nonrotating scattering surface. An application to torque measurement is then described in which maximum resistance to the effects of axial vibration is essential. Additional parameters that affect the performance of this proposed new instrument are also discussed.

\section{Theoretical Background}

It is convenient to consider only speckle intensity fluctuations, defined by $\Delta I(t)=I(t)-\overline{I(t)}$. The space-time correlation function $\Gamma_{\Delta I}(\Delta \mathbf{x}, \tau)$ of the speckle intensity fluctuation in the detecting plane can be given by

$$
\Gamma_{\Delta I}(\Delta \mathbf{x}, \tau)=\langle\Delta I(\mathbf{x}, t) \Delta I(\mathbf{x}+\Delta \mathbf{x}, t+\tau)\rangle,
$$

where $\mathbf{x}$ and $\Delta \mathbf{x}$ are, respectively, position and spatial delay in the detecting plane; $t$ and $\tau$ are, respectively, time and temporal delay; and $\langle\ldots\rangle$ denotes the ensemble average. If speckles are integrated over a detecting aperture, the integrated speckle intensity

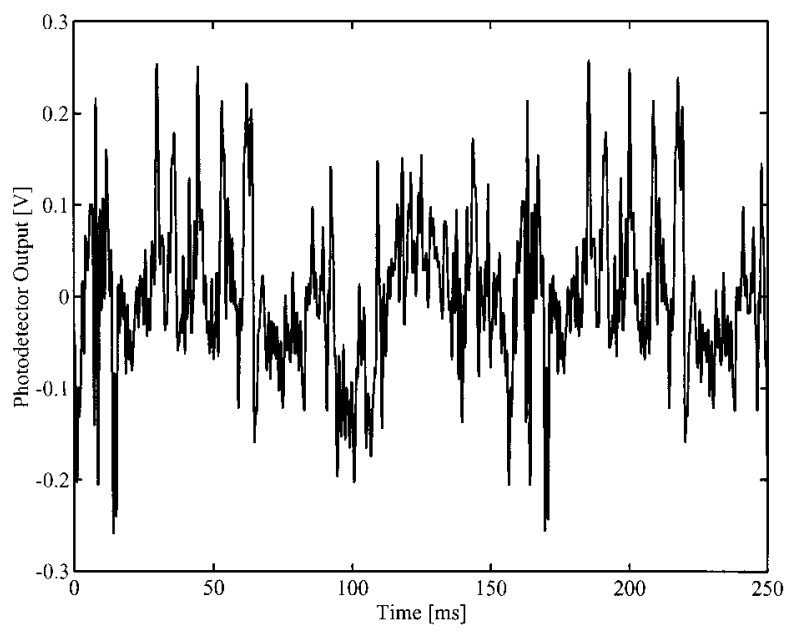

Fig. 2. Typical photodetector output.

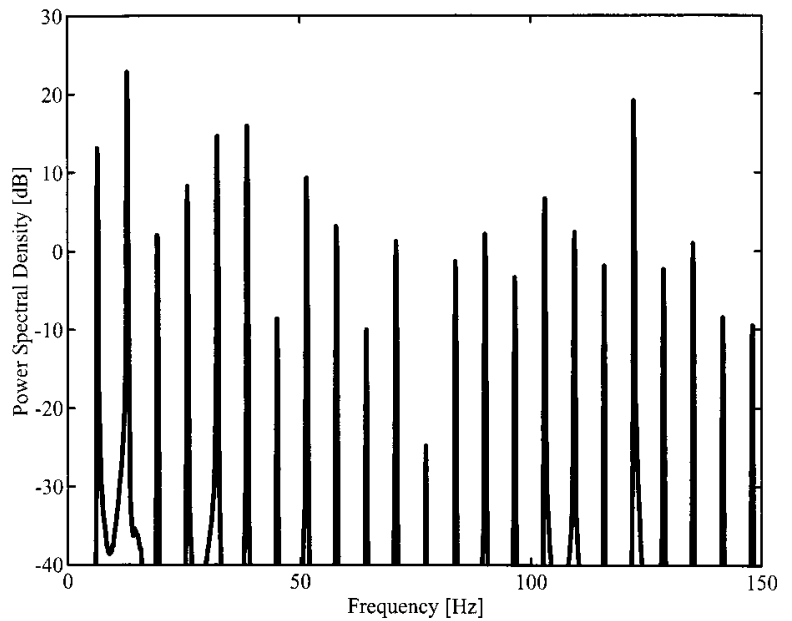

Fig. 3. Spectrum of photodetector output.

fluctuation space-time correlation function $\Gamma_{A}(\mathbf{X}, \tau)$ becomes

$$
\begin{aligned}
\Gamma_{A}(\mathbf{X}, \tau)= & \left\langle\left[\int_{-\infty}^{\infty} A(\mathbf{x}) \Delta I(\mathbf{x}, t) \mathrm{d} \mathbf{x} \int_{-\infty}^{\infty} A(\mathbf{x}+\Delta \mathbf{x}-\mathbf{X})\right.\right. \\
& \times \Delta I(\mathbf{x}+\Delta \mathbf{x}, t+\tau) \mathrm{d} \Delta \mathbf{x}]\rangle
\end{aligned}
$$

where $A(\mathbf{x})$ is the aperture function. In Eq. (2) each integral describes the summation of speckle intensity across the detector active area. The quantity $\mathbf{X}=$ $(X, Y)$ describes the spatial delay between the position of a detecting aperture at time $t$ and the position of a detecting aperture at time $(t+\tau)$. Introducing this delay into the aperture function leads to a spacetime correlation function rather than simply a time correlation function. Moving the ensemble average within the integral and reordering the integration result in a useful expression for the spatially integrated correlation function:

$$
\begin{aligned}
\Gamma_{A}(\mathbf{X}, \tau)= & \int_{-\infty}^{\infty}\left[\int_{-\infty}^{\infty} A(\mathbf{x}) A(\mathbf{x}+\Delta \mathbf{x}-\mathbf{X}) \mathrm{d} \mathbf{x}\right] \\
& \times\langle\Delta I(\mathbf{x}, \tau) \Delta I(\mathbf{x}+\Delta \mathbf{x}, t+\tau)\rangle \mathrm{d} \Delta \mathbf{x} .
\end{aligned}
$$

The inner bracketed term is recognized as the spatial autocorrelation of the aperture function as a function of $(\Delta \mathbf{x}-\mathbf{X})$. If the aperture is rectangular with dimensions $L_{x}$ by $L_{y}$, then the autocorrelation function of the aperture is a product of triangle functions. Asakura and Takai $^{1}$ validate the use of a Gaussian soft aperture as an approximation of a hard aperture. In the analysis presented here a Gaussian expression is used to approximate the autocorrelation function of the aperture rather than the aperture itself. An appropriate equation for the aperture autocorrelation 


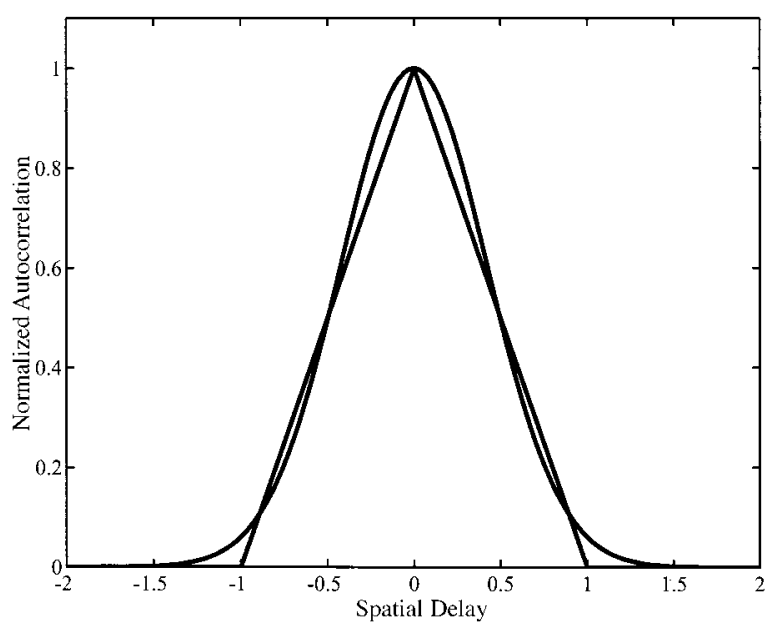

Fig. 4. Gaussian approximation of the aperture autocorrelation function and the actual triangular autocorrelation function of the aperture.

function, with the vector $\Delta \mathbf{x}-\mathbf{X}$ expanded as $(\Delta x-$ $X, \Delta y-Y)$, is
Setting $\tau=0$ in Eq. (5) and finding the $e^{-2}$ width of the correlation function gives an estimate of the speckle size $\left\langle\sigma_{0}\right\rangle$ in the detecting plane as

$$
\left\langle\sigma_{0}\right\rangle=\frac{2 \sqrt{2}}{\pi} \frac{\lambda z}{D} .
$$

When Eqs. (3)-(6) are combined, the resulting normalized correlation function can be written as

$$
\begin{aligned}
\gamma_{A}(X, Y, \tau)= & \frac{\Gamma_{A}(X, Y, \tau)}{\Gamma_{A}(0,0,0)}=\exp \left\{-\left[\left(\frac{2}{D}\right)^{2}+2\left(\frac{\rho}{\left\langle\sigma_{0}\right\rangle}\right)^{2}\right]\right. \\
& \left.\times\left(v_{\xi}{ }^{2}+v_{\eta}{ }^{2}\right) \tau^{2}\right\} \exp \left\{-k_{0}\left[\left(\frac{X}{L_{x}}\right)^{2}+\left(\frac{Y}{L_{y}}\right)^{2}\right]\right\} \\
& \times \exp \left[\frac{\left(k_{0}\left\langle\sigma_{0}\right\rangle^{2} X+2 \rho L_{x}{ }^{2} \nu_{\xi} \tau\right)^{2}}{L_{x}{ }^{2}\left\langle\sigma_{0}\right\rangle^{2}\left(k_{0}\left\langle\sigma_{0}\right\rangle^{2}+2 L_{x}{ }^{2}\right)}\right] \\
& \times \exp \left[\frac{\left(k_{0}\left\langle\sigma_{0}\right\rangle^{2} Y+2 \rho L_{y}{ }^{2} \nu_{\eta} \tau\right)^{2}}{L_{y}{ }^{2}\left\langle\sigma_{0}\right\rangle^{2}\left(k_{0}\left\langle\sigma_{0}\right\rangle^{2}+2 L_{y}{ }^{2}\right)}\right]
\end{aligned}
$$

Eq. (7) describes the normalized space-time correlation function of the spatially integrated speckle in-

$$
\int_{-\infty}^{\infty} A(\mathbf{x}) A(\mathbf{x}+\Delta \mathbf{x}-\mathbf{X}) \mathrm{d} \mathbf{x}=L_{x} \Lambda\left(\frac{\Delta x-X}{L_{x}}\right) L_{y} \Lambda\left(\frac{\Delta y-Y}{L_{y}}\right) \approx L_{x} \exp \left[-k_{0}\left(\frac{\Delta x-X}{L_{x}}\right)^{2}\right] L_{y} \exp \left[-k_{0}\left(\frac{\Delta y-Y}{L_{y}}\right)^{2}\right] .
$$

A numerical analysis was used to calculate the total square error between the actual aperture autocorrelation function and its Gaussian approximation. The calculation was performed across a widening range of spatial delay from the aperture width to a range much wider than the actual aperture width. For a rectangular aperture the value of $k_{0}$ for the minimum error converges quickly to 2.85 . The approximation of the Gaussian aperture autocorrelation function is indicated in Fig. 4.

The ensemble average within the outer integral in Eq. (3) is recognized as the speckle intensity fluctuation correlation function in Eq. (1), and this is given by $^{1}$

$$
\begin{aligned}
\Gamma_{\Delta I}(\Delta \mathbf{x}, \tau)= & c \exp \left(-\frac{4|\mathbf{v}|^{2} \tau^{2}}{D^{2}}\right) \\
& \times \exp \left[-\left(\frac{\pi D}{2 \lambda z}\right)^{2}|\Delta \mathbf{x}-\rho \mathbf{v} \tau|^{2}\right],
\end{aligned}
$$

where $c$ is a scaling constant, $\mathbf{v}$ is a vector describing the velocity of the scatterers in the $(\xi, \eta)$ plane, $D$ is the diameter of the illuminating beam, $\lambda$ is the laser wavelength, $z$ is the target-detector separation, and $\rho$ is a wave-front curvature term given by $1+z / r$, where $r$ is the wave-front curvature radius of the illuminating beam. (The sign notation with respect to the wave-front curvature is applied in the following way: $r$ is positive when the beam waist is to the left of the wave front.) tensity backscattered from a target with in-plane motion. This proves to be a useful expression to study the effect of various target motions on the photodetector output signal.

\section{A. Target Rotation}

For a rotating target both the tilt of the illuminated surface as it rotates and the tangential velocity associated with rotation must be accounted for. For analysis of the effect of target rotation, Eq. (7) can be written in terms of a rotation angle $\theta=\omega \tau$. The rotational decorrelation angle $\theta_{c}$ is defined as the rotation angle at which the resulting normalized autocorrelation falls to $e^{-2}$. The surface tilts at the same rate at which it rotates, $\omega$, giving rise to speckle translation in the detecting plane at a velocity of $2 \omega z .^{2}$ In Eq. (7) this is modeled as a photodetector displacement $X=0, Y=2 \omega z \tau$, whereas the tangential velocity of the shaft of radius $R$ is modeled by setting $v_{\eta}=R \omega$. Neglecting motion in the $\xi$ direction, one can determine the rotational decorrelation angle by manipulating Eq. (7):

$$
\theta_{c}=\sqrt{2}\left[\left(\frac{2 R}{D}\right)^{2}+\frac{2 k_{0}(2 z+\rho R)^{2}}{k_{0}\left\langle\sigma_{0}\right\rangle^{2}+2 L_{y}^{2}}\right]^{-1 / 2}
$$

Equation (8) for this special case is in agreement with that derived previously by an alternative method. ${ }^{3}$ The decorrelation angle is important in a later consideration of the photodetector outputs in the proposed laser torquemeter. 


\section{B. Resistance to Decorrelation Caused by In-Plane Target Displacement}

For the main purpose of this study, which is the decorrelation of the photodetector output caused by axial motion at velocity $\nu_{\xi}$, the target rotation can be neglected, $\omega=0$, and in this special case Eq. (7) reduces to

$$
\gamma_{A}(\tau)=\exp \left\{-\left[\left(\frac{2}{D}\right)^{2}+\frac{2 k_{0} \rho^{2}}{k_{0}\left\langle\sigma_{0}\right\rangle^{2}+2 L_{x}^{2}}\right] \nu_{\xi}^{2} \tau^{2}\right\} .
$$

If the decorrelation displacement $x_{c}=v_{\xi} \tau_{c}$ is defined as the axial displacement required to reduce the normalized autocorrelation to $e^{-2}$, then $x_{c}$ is given by the following:

$$
\frac{x_{c}}{D}=\left(\frac{k_{0}\left\langle\sigma_{0}\right\rangle^{2}+2 L_{x}^{2}}{2 k_{0}\left\langle\sigma_{0}\right\rangle^{2}+4 L_{x}^{2}+k_{0} \rho^{2} D^{2}}\right)^{1 / 2} .
$$

This ratio between decorrelation displacement and beam diameter, $x_{c} / D$, is proposed as a measure of the effectiveness of a configuration in resisting decorrelation. Importantly, the ratio can be seen to take a maximum value of $1 / \sqrt{2} \approx 0.7$ when

$$
\left(2 k_{0}\left\langle\sigma_{0}\right\rangle^{2}+4 L_{x}^{2}\right) \gg k_{0} \rho^{2} D^{2} .
$$

This inequality dictates an optimum combination of, effectively, beam diameter, speckle size, and photodetector size (in the direction of speckle motion) for which the maximum resistance to decorrelation of the photodetector output can be achieved.

\section{Experimental Measurement of Autocorrelation Function}

The most obvious way to acquire data to validate Eq. (10) is to translate the shaft axially relative to the illuminating beam and autocorrelate the resulting signal. This process needs to be repeated for each angular position of the shaft; however, there is a more efficient method of data acquisition that takes advantage of the shaft's rotation and the resulting signal's periodicity.

The matrix $\mathscr{S}$ summarizes the experimental data acquired when the laser beam illuminates the rotating shaft. In the matrix $\mathscr{Y}$ each column corresponds to the integrated intensity variation with axial position at a given angular location, and each row corresponds to the integrated intensity variation with angular position at a fixed axial position:

$$
\mathscr{Y}=\left[\begin{array}{cccccccc}
s_{11} & s_{12} & s_{13} & s_{14} & \cdots & s_{1 n} & \cdots & s_{1 N} \\
s_{21} & s_{22} & s_{23} & s_{24} & \cdots & s_{2 n} & \cdots & s_{2 n} \\
\cdot & \cdot & \cdot & \cdot & s_{m n} & \cdot & \cdots & \cdot \\
s_{M 1} & s_{M 2} & s_{M 3} & s_{M 4} & \cdots & \cdot & \cdots & s_{M N}
\end{array}\right] .
$$

One fills the matrix most efficiently by recording the photodetector output from (at least) one full shaft rotation at each axial position. In this way only a small number of data captures, at an appropriate sample rate, produces a matrix with approximately 20,000 columns. The matrix $\mathscr{S}$ is quickly filled row by row.
With data acquired in this manner cross correlation between rows is more convenient than autocorrelation of columns. In what follows, the equivalence of these two functions is shown.

To confirm the theoretical relation of maximum resistance to decorrelation, autocorrelation of the columns is required to determine the axial decorrelation displacement $x_{c}$. The experimental value of $x_{c}$ is found from the mean $e^{-2}$ width of the autocorrelation of every column in matrix $\mathscr{S}$. The discrete autocorrelation for the $n$th column as a function of axial delay $a$ is written $\Gamma_{n n}(a)$, where

$$
\Gamma_{n n}(a)=\sum_{m=1}^{M} s_{m n} s_{(m+a) n} .
$$

The mean of the discrete autocorrelations of $N$ columns can be written as

$$
\bar{\Gamma}_{n n}(a)=\frac{1}{N} \sum_{n=1}^{N} \sum_{m=1}^{M} s_{m n} s_{(m+a) n} .
$$

This method is sensitive to the synchronization of the rows of data. It is useful to consider the rows of matrix $\mathscr{Y}$ to be aligned, but the rows of experimental data need not be (and were not) aligned when captured by this method. The peak value of the cross correlation of a row with an arbitrarily chosen reference row is representative of the degree of similarity between the data in these two rows. With data acquired in this format, the cross correlation is a straightforward function to evaluate and its peak value is not dependent on the synchronization of the data. The cross correlation between row $m$ and row $(m+a)$, written $\Gamma_{m(m+a)}(\theta)$, has its peak value at zero angular delay, $\theta=0$, and this can be written as

$$
\Gamma_{m(m+a)}(0)=\sum_{n=1}^{N} s_{m n} s_{(m+a) n} .
$$

Averaging over $M$ cross correlations of rows separated by axial delay $a$,

$$
\bar{\Gamma}_{m(m+a)}(0)=\frac{1}{M} \sum_{m=1}^{M} \sum_{n=1}^{N} s_{m n} s_{(m+a) n} .
$$

The original aim was to estimate the mean discrete autocorrelation of $N$ columns, but this method of data acquisition is better suited to calculation of the crosscorrelation function. These two functions can now be related by use of Eqs. (14) and (16):

$$
\bar{\Gamma}_{n n}(a)=\frac{M}{N} \bar{\Gamma}_{m(m+a)}(0),
$$

and the mean discrete normalized autocorrelation is given by

$$
\bar{\gamma}_{n n}(a)=\frac{\bar{\Gamma}_{n n}(a)}{\bar{\Gamma}_{n n}(0)}=\frac{\bar{\Gamma}_{m(m+a)}(0)}{\bar{\Gamma}_{m m}(0)} .
$$

Thus the normalized autocorrelation of the integrated speckle intensity as a function of axial displacement (i.e., down the columns of $\mathscr{Y}$ ) is estimated 
conveniently as the peak value of the normalized cross correlation between the rows of $\mathscr{Y}$ as a function of axial delay. Since the cross-correlation peak values are not affected by the synchronization of the rows of $\mathscr{Y}$, there is no need to realign the rows in this analysis. This would, of course, be necessary to calculate the autocorrelations of each column of $\mathscr{Y}$ directly.

\section{A. Experimental Research}

A shaft of $4.35-\mathrm{cm}$ radius coated with retro-reflective tape was prepared as a rotating target. The shaft was driven by a brushless dc motor with a rotational frequency of $6.4 \mathrm{~Hz}$ and was illuminated by a $5-\mathrm{mW}$ $\mathrm{He}-\mathrm{Ne}$ laser of 632.8-nm wavelength. A photodetector was positioned with its center coincident with the optical axis so that the mean output signal was maximized. Time-varying speckle intensity was detected by the photodetector and captured on a digital oscilloscope. A signal, from one revolution of the shaft, was captured and downloaded to a computer. This signal, consisting of $N$ samples, corresponds to one row of matrix $\mathscr{Y}$. The shaft was then displaced in discrete steps in the axial direction by a linear translation stage until the total displacement exceeded the $e^{-2}$ diameter of the beam illuminating the shaft. For each of the displaced axial shaft positions the data capture process was repeated, each time adding another row to matrix $\mathscr{Y}$.

A middle row of matrix $\mathscr{Y}$ was selected as a reference signal, and all the other rows were cross correlated with this reference signal. The normalized autocorrelation function was estimated from Eq. (18) and plotted versus the axial distance from the reference position. A Gaussian curve was then fitted to the set of peak values to simplify the estimate of the axial decorrelation displacement. The whole experiment was repeated and $\bar{\gamma}_{n n}$ plotted with error bars indicating \pm 1 standard deviation. Figures 5(a)-5(c) show typical plots $\bar{\gamma}_{n n}$ against axial displacement (product of delay $a$ and spatial increment between measurements).

\section{B. Discussion}

An intuitive approach leads one to expect $x_{c}$ to increase with an increase in beam diameter because of the large number of illuminated scatterers. Shaft translation results in a small proportion of scattering elements being lost and proportionally only a few new scattering elements being gained, so the resulting speckle pattern is essentially unchanged. A similar argument can be presented concerning speckle size: a few large speckles on the detector result in the photodetector signal decorrelating when the shaft is displaced because the speckle intensity is significantly changed by the disappearance and appearance of individual speckles on the detector.

Table 1 summarizes the optical configurations for which the resistance to decorrelation of the photodetector output was investigated. The configurations are listed in ascending order of $L_{x} /\left\langle\sigma_{0}\right\rangle$. Table 1 also indicates likely configurations for practical setups,
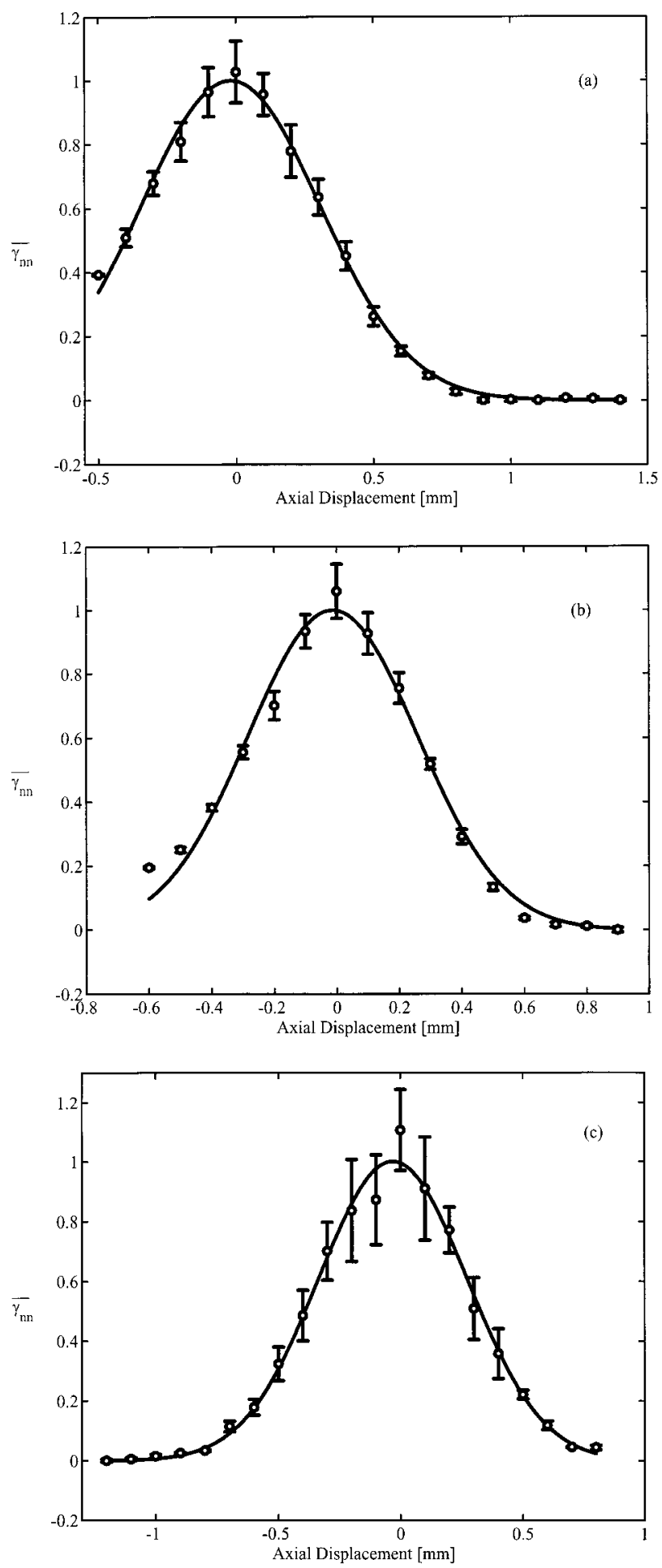

Fig. 5. Normalized autocorrelation as a function of axial displacement: (a) $z=30 \mathrm{~cm}, D=1.45 \mathrm{~mm}, x_{c}=0.62 \mathrm{~mm}$; (b) $z=16 \mathrm{~cm}$, $D=1.18 \mathrm{~mm}, x_{c}=0.54 \mathrm{~mm}$; (c) $z=57 \mathrm{~cm}, D=1.45 \mathrm{~mm}, x_{c}=0.64$ $\mathrm{mm}$.

particularly configurations for which $\left(x_{c} / D\right)_{\max } \approx 0.7$ is achieved. Axial displacements greater than $0.7 D$ cannot be accommodated, for example, by increasing the detector size, as the scatterers and the resultant speckle patterns are now too different from those that 


\begin{tabular}{|c|c|c|c|c|c|c|}
\hline Data Point & $L_{x} /\left\langle\sigma_{0}\right\rangle$ & $\mathrm{x}_{\mathrm{c}} / \mathrm{D}$ & $\begin{array}{c}D \\
(\mathrm{~mm})\end{array}$ & $\begin{array}{c}z \\
(\mathrm{~cm})\end{array}$ & $\begin{array}{c}L_{x} \\
(\mathrm{~mm})\end{array}$ & $\begin{array}{c}L_{y} \\
(\mathrm{~mm})\end{array}$ \\
\hline$a$ & 2.1 & 0.355 & 1.45 & 107.0 & 1.0 & 1.0 \\
\hline $\mathrm{b}$ & 3.9 & 0.372 & 1.45 & 57.0 & 1.0 & 1.0 \\
\hline$f$ & 5.29 & 0.327 & 1.94 & 57.0 & 1.0 & 1.0 \\
\hline $\mathrm{g}$ & 6.8 & 0.269 & 2.51 & 57.0 & 1.0 & 1.0 \\
\hline $\mathrm{h}$ & 11.2 & 0.206 & 3.54 & 49.0 & 1.0 & 1.0 \\
\hline$d$ & 13.3 & 0.420 & 1.45 & 17.0 & 1.0 & 1.0 \\
\hline $\mathrm{c}$ & 21.4 & 0.476 & 1.18 & 16.0 & 1.0 & 1.0 \\
\hline $\mathrm{e}$ & 28 & 0.721 & 0.882 & 30.0 & 5.9 & 1.1 \\
\hline $\mathrm{i}$ & 44 & 0.716 & 1.43 & 30.0 & 5.9 & 1.1 \\
\hline
\end{tabular}

created the original set of speckle patterns. A general trend in the data indicates increasing $x_{c} / D$ with increasing $L_{x} /\left\langle\sigma_{0}\right\rangle$, but this relationship is also influenced by beam diameter, which acts, not surprisingly, to reduce $x_{c} / D$. Ideally, optimum performance, $\left(x_{c} / D\right) \approx 0.7$, is required, suggesting use of a small beam diameter, but large $x_{c}$ in absolute terms is also desirable, which requires a large beam diameter. In reality, a beam diameter is chosen based on knowledge of the expected axial motion, and optimum performance is then ensured by the appropriate choice of optical configuration. These experimental observations support Eq. (10), but agreement with theoretical analysis is best illustrated by Fig. 6 , which shows excellent agreement in a comparison between the experimental and theoretical values of $x_{c}$. (The data points in Fig. 6 and Table 1 are labeled with the letters a-i to facilitate comparison.)

\section{Implications for the Laser Torquemeter}

The laser torquemeter ${ }^{4}$ is an instrument that measures time-resolved torque by noncontacting means. Two independent laser beams are incident on a rotating shaft and are axially separated by a known distance $\Delta L$. Independent photodetectors are associated with each illuminating beam, and their output signals (with the mean removed by ac coupling) are

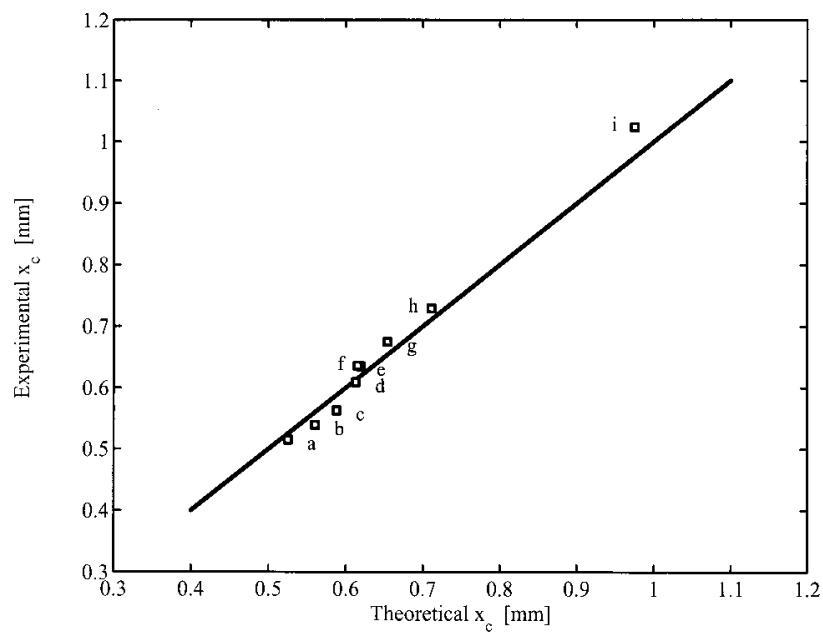

Fig. 6. Plot of theoretical $x_{c}$ against experimental $x_{c}$. digitally stored into the memory for one revolution of the shaft. During measurement these stored signals are electronically locked with the current signals from each photodetector to determine the angular position of the shaft. As torsional load is applied to the shaft, the relative phase of each pair of recorded and current waveforms varies in proportion to the twist of the shaft, which is directly proportional to torque. To calculate the instantaneous torque on a shaft, the laser torquemeter needs to track the shaft twist from when the zero-torque reference signal is stored into memory to when the load of interest is fully applied. If the stored reference signal varies significantly from the current photodetector signal, the lock between recorded and current signals is lost and tracking of shaft twist becomes impossible, preventing further torque measurement. The load needs to be removed and the zero-torque reference signal stored again. For reliable operation the laser torquemeter needs to be tolerant of vibrations that disturb the correlation between the reference signal and the current signal.

\section{A. Maximizing Resistance to Decorrelation Caused by Axial Shaft Vibration}

Choice of the principal adjustable parameters $z, D$, and $L_{x}$ is made by considering the expected axial vibration displacement. The axial vibration of the driven shaft on the output of a 4-cylinder diesel engine was found to be of the order of $1 \mathrm{~mm}$ peak to peak, and from this measurement the minimum illuminating beam diameter can be determined. The beam diameter must be large enough to avoid decorrelation of the signals but at the same time small, relative to axial separation, to avoid uncertainty in the value of $\Delta L$ that is required to calculate torque from the twist measurement. Significant shaft twist across the beam diameter might also result in decorrelation of the signals, but the shaft twists encountered in practice are unlikely to be sufficiently large.

Equation (10) suggests that the minimum illuminating beam diameter should be at least $\sqrt{2}$ of the axial vibration displacement of the shaft. Experience with the prototype laser torquemeter suggests that the normalized correlation between the recorded signal and the current photodetector output signal 
can fall only to approximately 0.5 , rather than $e^{-2}$, before the lock between the signals is lost. This suggests that the minimum illuminating beam diameter should be at least 2.5 times the axial vibration displacement of the shaft. For maximum effectiveness in resisting decorrelation of the photodetector output, at a convenient target-detector separation $z$, inequality (11) suggests that $L_{x}$ must be well in excess of 2 $\mathrm{mm}$. A photodetector with $L_{x}$ of $5 \mathrm{~mm}$, for example, positioned with $z=10 \mathrm{~cm}$, would give $90 \%$ of the maximum achievable resistance to decorrelation.

\section{B. Controlling the Angular Correlation}

For adequate resolution of angle in the photodetector output signals and therefore shaft twist in the laser torquemeter output, use of signals with approximately 2000 fluctuations per revolution of the shaft, i.e., $\theta_{c}=2 \pi / 2000 \mathrm{rad}$, was found to be convenient in the prototype instrument. For the free-space optical configurations used in this study, typical shaft radii in the range from 2 to $10 \mathrm{~cm}$, a convenient targetdetector separation of $10 \mathrm{~cm}$, and an illuminating beam diameter of $2.5 \mathrm{~mm}$, as described in Subsection 4.A, Eq. (8) indicates a suitable value of $L_{y}=1 \mathrm{~mm}$. Note that $L_{x}$ has no influence on this correlation angle, but it does have an important effect on the modulation depth of the photodetector output signal, and this is addressed in Section 4.D.

\section{Effect of Radial $(\eta)$ Motion}

The photodetector output signal is also sensitive to radial $(\eta)$ motion but to a lesser extent than axial $(\xi)$ motion, which has been the main focus here. This is because the speckle motion resulting from the rotation of the shaft bears some similarity to the speckle motion resulting from the radial motion of the shaft. If changes in the backscattered speckle patterns were the same in each of the above cases, then the normalized cross correlation between one cycle of the photodetector output and a second cycle for which the position of the incident beam had been raised in the $(\eta)$ direction would show merely a change in the position of the cross-correlation peak without any change in its amplitude.

Figure 7 shows the way the normalized cross correlation varies as the incident beam is moved in the ( $\eta$ direction for one optical configuration. The shift in the position of the cross-correlation peak is clear, but at the same time there is a significant reduction in the amplitude of the peak. This shows that the photodetector output is influenced by the change in the position of the incident beam in the $(\eta)$ direction, i.e., the change in the backscattered speckle patterns is not merely a delay or advance in the cycle of the continuously changing speckle pattern. In particular, observation of the speckle pattern backscattered from a nonrotating shaft suggests that the changing shape of the surface at the point of illumination encourages boiling of the speckle pattern, compared with the scattering from a flat surface. Experimentation suggests that, as a result, increasing $L_{y}$ cannot be used as a means to resist decorrelation in the

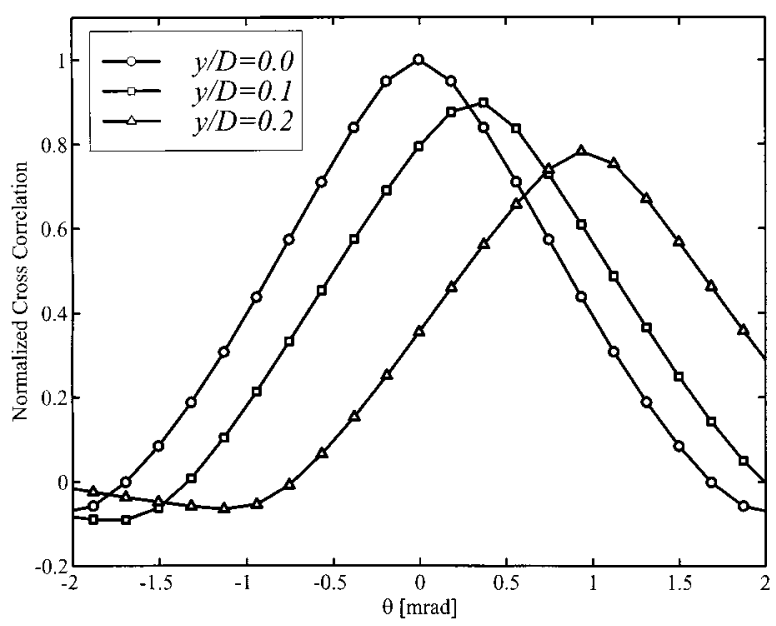

Fig. 7. Plot of the normalized cross-correlation function for $y / D=$ $0,0.1$, and $0.2: \quad D=1 \mathrm{~mm}, z=28 \mathrm{~cm}, L_{y}=1 \mathrm{~mm}$.

photodetector output. Initial experimentation suggests that maximization of the beam radius of curvature and the shaft radius might be beneficial. Use of an elliptical beam will also be the subject of further research.

\section{Modulation Depth in the Photodetector Output}

The modulation depth in the photodetector output signal is defined as the ratio of the standard deviation in the output voltage to the mean output voltage. It is advantageous to maximize modulation depth from the points of view of both photodetector noise and the processing of the signal within the laser torquemeter. Goodman has described a rms signal-to-noise ratio ${ }^{5}$ that is, in fact, the reciprocal of the modulation depth described above. This reference indicates that maximum modulation depth is achieved when the speckle size is greater than or equal to the photodetector size. The configuration demanded by the need to resist decorrelation caused by axial shaft vibration means that the maximum possible modulation depth cannot be achieved. The clear implication for the laser torquemeter is that $L_{x}$ should be as small as possible within the constraints set by axial shaft vibration.

\section{Conclusions}

When a laser beam is incident on a rotating diffuse object and the backscattered speckle patterns are sampled by a suitably positioned photodetector, the photodetector output signal is periodic. We have investigated the integrated speckle intensity cross correlation between a reference photodetector output signal and a photodetector output signal taken after axial displacement of the rotating shaft. It has been shown that the decorrelation displacement $x_{c}$ increases in a nonlinear fashion with both the beam diameter $D$ and the number of speckles collected. The ratio $x_{c} / D$ has a maximum value of approximately 0.7 , and this has been predicted theoretically and confirmed experimentally.

Using an efficient method of data acquisition that takes advantage of the shaft rotation and the result- 
ing periodicity of the photodetector output, we verified the theoretical prediction. The quality of this verification was enhanced by the evaluation process, in which convenient calculation of the peak of the cross-correlation function was used to calculate the required autocorrelation function as a mean of, effectively, several thousand independent autocorrelations.

The importance of this behavior has been described with reference to a proposed laser torquemeter, but wide applicability exists in situations in which speckle decorrelation is either desirable or undesirable. The theory and its validation are also equally suited to the examination of speckle patterns scattered from nonrotating targets undergoing in-plane motions.

The requirement to resist decorrelation of the photodetector output caused by shaft axial motion was shown to dictate a minimum incident beam size in the axial direction and a minimum photodetector size in the axial direction. The additional requirement to measure the separation of the two beams within the laser torquemeter, for calculating shaft torque from shaft twist, fixes the optimum beam size at the minimum necessary to resist shaft axial motion. An additional requirement to maximize the modulation depth of the photodetector output signal fixes the optimum $L_{x}$, for practical optical configurations, at the minimum necessary to resist decorrelation caused by axial shaft motion. In the radial $(\eta)$ direction the beam extent and the photodetector dimension are important in controlling the number of fluctuations in one period of the photodetector output. This is a compromise between the resolution in the twist measurement and the maintenance of the lock between the recorded and current signals.

\section{References}

1. T. Asakura and N. Takai, "Dynamic laser speckles and their application to velocity measurements of the diffuse object," Appl. Phys. 25, 179-194 (1981).

2. N. George, "Speckle from rough moving objects," J. Opt. Soc. Am. 66, 1182-1194 (1976).

3. N. Takai, T. Iwai, and T. Asakura, "An effect of curvature of rotating diffuse objects on the dynamics of speckles produced in the diffraction field," Appl. Phys. B (Photophys. Laser Chem.) 26, 185-192 (1981).

4. T. H. Wilmshurst, S. J. Rothberg, and N. A. Halliwell, "Laser torquemeter: a new instrument," Electron. Lett. 27, 186-187 (1991).

5. J. W. Goodman, "Statistical properties of laser speckle patterns," in Laser Speckle and Related Phenomena, J. C. Dainty, ed. (Springer-Verlag, Berlin, 1975), pp. 9-75. 